\title{
A study of phase noise in colpitts and LC-tank CMOS oscillators
}

\author{
Andreani, Pietro; Wang, Xiaoyan; Vandi, Luca; Fard, A.
}

Published in:

I E E E Journal of Solid State Circuits

Link to article, DOI:

10.1109/JSSC.2005.845991

Publication date:

2005

Document Version

Publisher's PDF, also known as Version of record

Link back to DTU Orbit

Citation $(A P A)$ :

Andreani, P., Wang, X., Vandi, L., \& Fard, A. (2005). A study of phase noise in colpitts and LC-tank CMOS oscillators. I E E E Journal of Solid State Circuits, 4O(5), 1107-1118. https://doi.org/10.1109/JSSC.2005.845991

\section{General rights}

Copyright and moral rights for the publications made accessible in the public portal are retained by the authors and/or other copyright owners and it is a condition of accessing publications that users recognise and abide by the legal requirements associated with these rights.

- Users may download and print one copy of any publication from the public portal for the purpose of private study or research.

- You may not further distribute the material or use it for any profit-making activity or commercial gain

- You may freely distribute the URL identifying the publication in the public portal

If you believe that this document breaches copyright please contact us providing details, and we will remove access to the work immediately and investigate your claim. 


\title{
A Study of Phase Noise in Colpitts and $L C$-Tank CMOS Oscillators
}

\author{
Pietro Andreani, Member, IEEE, Xiaoyan Wang, Luca Vandi, and Ali Fard
}

\begin{abstract}
This paper presents a study of phase noise in CMOS Colpitts and $\boldsymbol{L C}$-tank oscillators. Closed-form symbolic formulas for the $1 / f^{2}$ phase-noise region are derived for both the Colpitts oscillator (either single-ended or differential) and the $L C$-tank oscillator, yielding highly accurate results under very general assumptions. A comparison between the differential Colpitts and the $L C$-tank oscillator is also carried out, which shows that the latter is capable of a 2-dB lower phase-noise figure-of-merit (FoM) when simplified oscillator designs and ideal MOS models are adopted.

Several prototypes of both Colpitts and $L C$-tank oscillators have been implemented in a $0.35-\mu \mathrm{m}$ CMOS process. The best performance of the $L C$-tank oscillators shows a phase noise of $-142 \mathrm{dBc} / \mathrm{Hz}$ at 3-MHz offset frequency from a $2.9-\mathrm{GHz}$ carrier with a 16-mW power consumption, resulting in an excellent FoM of $\sim 189 \mathrm{dBc} / \mathrm{Hz}$. For the same oscillation frequency, the FoM displayed by the differential Colpitts oscillators is $\sim 5 \mathrm{~dB}$ lower.
\end{abstract}

Index Terms-CMOS, Colpitts, LC-tank, oscillators, phase noise.

\section{INTRODUCTION}

$\mathbf{T}$ $\mathrm{HE}$ continuing massive research production in the area of integrated radio-frequency (RF) voltage-controlled oscillators (VCOs) has probably not escaped the attention of any JSSC reader with but a superficial interest in RF applications. Such a focus on VCOs is certainly not unwarranted, as they still are the performance bottleneck in many radio receiver/transmitter designs.

While the classical differential $L C$-tank oscillator, in one or another of its many variants, has become the standard choice in the RF community, the differential Colpitts oscillator has also been the subject of several recent works (see e.g., [1], [2], [4], [5], [3]), in the reasonable attempt of extending the outstanding phase-noise performances of single-ended Colpitts oscillators to differential versions as well.

Although the excellent behavior of the Colpitts oscillator had already been experimentally recognized several decades ago, the first theoretical explanation for it had to await Hajimiri's and Lee's relatively recent seminal paper on phase noise [6]. In [6], the very low Colpitts phase noise was traced back to the weak impact that the noise from the transistor had on the

Manuscript received July 13, 2004; revised January 25, 2005

P. Andreani and L. Vandi are with the Center for Physical Electronics, Ørsted DTU, Technical University of Denmark, DK-2800 Kgs. Lyngby, Denmark (e-mail: pa@oersted.dtu.dk; 1v@oersted.dtu.dk).

X. Wang was with the Center for Physical Electronics, Ørsted DTU, Technical University of Denmark, DK-2800 Kgs. Lyngby, Denmark. She is now with Infineon Technologies AG, D-81677 Munich, Germany (e-mail: Xiaoyan.Wang1@infineon.com).

A. Fard is with the Department of Electronics, Mälardalen University, SE-72123 Västerås, Sweden (e-mail: ali.fard@ @mdh.se).

Digital Object Identifier 10.1109/JSSC.2005.845991 phase noise itself. Central in Hajimiri's approach is the concept of Impulse Sensitivity Function (ISF, with symbol $\Gamma$ ), whose lesson is that the impact of any noise source on the oscillator phase noise varies across the oscillation period. This key insight can be rephrased in the following way: the same amount of noise generates a different level of phase noise, depending on when the noise source is active. An impressive example of this fact will be demonstrated in Section III.

Hajimiri was able to show that numerical results derived from the ISF theory matched well experimental results from a number of oscillators, including a single-ended Colpitts. A differential CMOS Colpitts oscillator design making partial use of a numerical ISF approach was presented in [4], while an analysis leading to a mixed numerical-symbolic phase noise equation for a single-ended CMOS Colpitts oscillator was undertaken in [7], where, however, the time-variant nature of the noise to phase-noise conversion was neglected.

The present work has two main goals: 1) to provide a closedform symbolic expression for the phase noise displayed by the CMOS Colpitts oscillator (either single-ended or differential, see Fig. 1) in the $1 / f^{2}$ region, making use of the ISF theory and 2) based on this result, to compare the phase-noise performance of the differential Colpitts oscillator with that of the more popular differential $L C$-tank oscillator, in order to ascertain whether it is justified to target the Colpitts oscillator as a better substitute for the $L C$-tank oscillator. ${ }^{1}$

The rest of this paper is organized as follows. Section II develops the symbolic phase-noise ISF analysis for the Colpitts oscillator, while Section III compares these results with those obtained when the time-variant nature of phase noise generation is neglected, evidencing the large error so introduced. Section IV derives in a rigorous way a closed-form equation for the $1 / f^{2}$ phase noise in LC-tank oscillators. The comparisons and considerations in Section $\mathrm{V}$ show that the $L C$-tank oscillator is the better one, at least with regards to performances in the $1 / f^{2}$ phase noise region; Section VI presents actual measurement results on both Colpitts and $L C$-tank oscillators, supporting the conclusions of Section V; and Section VII summarized this work's most important contributions.

\section{Phase-Noise ANALYSIS IN COLPITTS OSCILlATORS}

\section{A. Conduction Angle and Oscillation Amplitude}

The single-ended Colpitts oscillator under analysis is shown in Fig. 1(a), where resistance $R_{t}$ accounts for the losses in both

\footnotetext{
${ }^{1}$ The definition " $L C$-tank oscillator" is actually rather vague, since a Colpitts oscillator is of course a kind of $L C$ oscillator as well. Nevertheless, following the current praxis, we assume in this work that the $L C$-tank oscillator is the one in Fig. 2, and nothing else.
} 


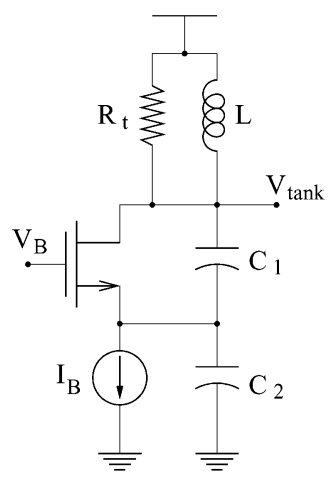

(a)

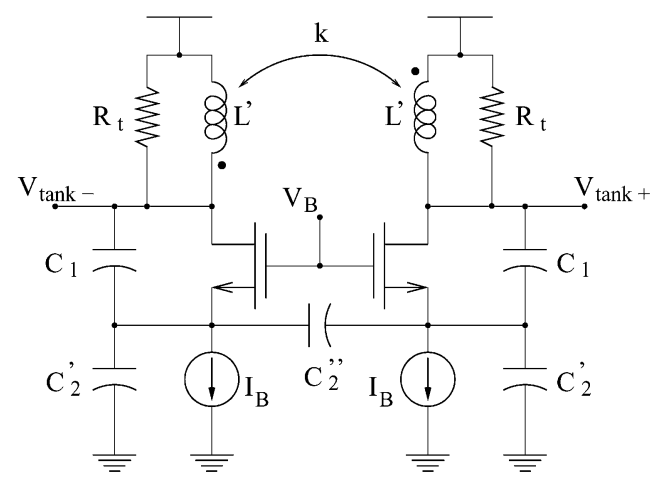

(b)

Fig. 1. (a) Single-ended Colpitts oscillator. (b) Differential Colpitts oscillator.

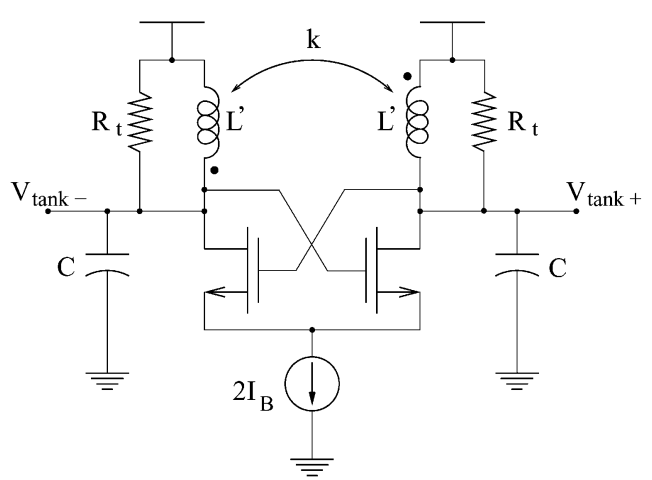

Fig. 2. $L C$-tank oscillator.

inductor and capacitors. We assume throughout this work that the ideal square-law relation between $I_{\mathrm{ds}}$ and $V_{\mathrm{gs}}$ describes the behavior of the MOS transistor. Although it is well-known that this assumption is optimistic in deep-submicron CMOS processes, it has the advantage of leading to manageable results, and, more importantly, results that can be taken as upper boundaries for the performances of the real Colpitts oscillator. The impact of second-order deviations from the square law will be qualitatively discussed in Section V.

We start our analysis with the consideration that, even for a moderately selective resonator, all transistor current harmonics higher than the fundamental are filtered off, and can be disregarded in the calculation of the voltage amplitude at the output of the oscillator. Assuming that the transistor loads $C_{2}$ only weakly, we can simplify the treatment by assuming that the ac voltage $V_{s}(t)$ at the source of the transistor is sinusoidal with amplitude $A_{s}$ and angular frequency $\omega_{0}$. Therefore, with arbitrary phase at $t=0$, we have

$$
V_{s}(t)=-A_{s} \cos \left(\omega_{0} t\right)
$$

or

$$
V_{s}(\phi)=-A_{s} \cos (\phi)
$$

where the angle $0 \leq \phi \leq 2 \pi$ is used instead of $\omega_{0} t$.

Calling $V_{\text {od }}$ the effective DC voltage between MOS gate and source, $I_{\mathrm{ds}}$ is written as ${ }^{2}$

$$
I_{\mathrm{ds}}(\phi)=\frac{\beta}{2}\left[-V_{\text {od }}+A_{s} \cos (\phi)\right]^{2}
$$

where $\beta=\mu_{e} C_{\mathrm{ox}} W / L$ ( $\mu_{e}$ being the electron mobility, $C_{\mathrm{ox}}$ the gate oxide capacitance per unit area, and $W$ and $L$ the transistor width and length, respectively), and $V_{\text {od }}$ is a DC-voltage. It is well known that the active device in the Colpitts oscillator works in a class- $\mathrm{C}$ manner, meaning that it delivers more or less narrow current pulses for a (small) fraction of the oscillation period, referred to as conduction angle, and is in the off-state for the rest of the period. It is immediate from (3) that class-C operations entail a positive value for $V_{\text {od }}$, while the limit between class $\mathrm{C}$ and class B is given by $V_{\mathrm{od}}=0$. Calling $\Phi$ half the conduction angle, for which $I_{\mathrm{ds}}$ becomes zero, we obtain from (3)

$$
\cos (\Phi)=\frac{V_{\mathrm{od}}}{A_{s}}
$$

and (3) becomes

$$
I_{\mathrm{ds}}(\phi)=\frac{\beta}{2} A_{s}^{2}[\cos (\phi)-\cos (\Phi)]^{2} .
$$

Obviously, the DC value for the MOS current must be equal to the bias current $I_{B}$; therefore, over one signal period we must have

$$
\begin{aligned}
I_{B} & =\frac{1}{2 \pi} \int_{-\Phi}^{\Phi} I_{\mathrm{ds}}(\phi) d \phi \\
& =\frac{\beta}{2 \pi} A_{s}^{2}\left[-\frac{3}{2} \cos (\Phi) \sin (\Phi)+\frac{\Phi}{2}+\Phi \cos ^{2}(\Phi)\right]
\end{aligned}
$$

which is very well approximated by

$$
I_{B} \approx \frac{\beta A_{s}^{2}}{15 \pi} \Phi^{5}\left(1-\frac{4}{21} \Phi^{2}\right) .
$$

Neglecting the higher order term, a simple and still quite accurate expression for small $\Phi$ values is

$$
\Phi \approx\left(\frac{15 \pi I_{B}}{\beta A_{s}^{2}}\right)^{\frac{1}{5}} .
$$

We proceed by calculating the first harmonics $I_{1}$ of the current generated by the transistor. By means of Fourier's theory, and considering that $I_{\mathrm{ds}}$ is an even function of $\phi$, we obtain

$$
\begin{aligned}
I_{1} & =\frac{1}{\pi} \int_{-\Phi}^{\Phi} I_{\mathrm{ds}}(\phi) \cos (\phi) d \phi \\
& =\frac{\beta}{\pi} A_{s}^{2}\left[\frac{1}{3} \cos ^{2}(\Phi) \sin (\Phi)+\frac{2}{3} \sin (\Phi)-\Phi \cos (\Phi)\right]
\end{aligned}
$$

${ }^{2}$ This first part of the analysis resembles quite closely Huang's [7], with the difference that we make an explicit use of the conduction angle $2 \Phi$, defined in (4). This choice results in very simple equations, and allows a direct estimation of the impact of the relative width of the current pulses on the oscillator behavior. 
which is simplified to

$$
I_{1} \approx 2 \frac{\beta A_{s}^{2}}{15 \pi} \Phi^{5}\left(1-\frac{11}{42} \Phi^{2}\right)
$$

Using (7) and (10), we are able to express $I_{1}$ as

$$
I_{1} \approx 2 I_{B}\left(1-\frac{\Phi^{2}}{14}\right) \text {. }
$$

To find the voltage amplitude generated by $I_{1}$ we follow the describing-function approach [8], where the large-signal transconductance $G_{m}$ of the transistor is calculated as $G_{m}=I_{\mathrm{ds}, \text { fund }} / V_{\mathrm{gs}, \text { fund }}, I_{\mathrm{ds}, \text { fund }}$ and $V_{\mathrm{gs}, \text { fund }}$ being the first harmonics of drain current and gate-source voltage, respectively. Using the actual values of $I_{\mathrm{ds}, \text { fund }}$ and $V_{\mathrm{gs}, \text { fund }}, G_{m}$ becomes

$$
G_{m}=-\frac{I_{1}}{A_{s}} .
$$

Assuming a high quality factor $Q$ for the resonator, it is straightforward to show [8], and will not be repeated here, that the use of (12) results in an angular frequency of oscillation

$$
\omega_{0}=\frac{1}{\sqrt{L C}}
$$

where

$$
C \equiv \frac{C_{1} C_{2}}{C_{1}+C_{2}}
$$

and in an $A_{s}$ equal to

$$
A_{s}=I_{1} R_{t}(1-n) n
$$

with

$$
n \equiv \frac{C_{1}}{C_{1}+C_{2}} .
$$

Further, the voltage $V_{\operatorname{tank}}(\phi)$ at the Colpitts output (see Fig. 1) is given by

$$
V_{\text {tank }}(\phi)=V_{s}(\phi) / n=-A_{\text {tank }} \cos (\phi)
$$

with peak amplitude

$$
A_{\text {tank }}=I_{1} R_{t}(1-n) .
$$

Through (11) and (18), $A_{\text {tank }}$ can be expressed as a function of bias current and conduction angle: ${ }^{3}$

$$
A_{\text {tank }}=2 I_{B} R_{t}(1-n)\left(1-\frac{\Phi^{2}}{14}\right)
$$

1) Single-Ended Versus Differential: Although the previous equations have been obtained in the single-ended case, it is straightforward to extend them to the differential Colpitts of Fig. 1(b), where a differential mode of operation is enforced either by coupling the two single-ended oscillators through the floating capacitor ${ }^{4} C_{2}^{\prime \prime}$, or, new in this work, by coupling

${ }^{3}$ Equation (19) yields $A_{\text {tank }}$ in an implicit way, since $\Phi$ is itself a function of $A_{s}$, and consequently of $A_{\text {tank }}$. However, considering that the dependence of $A_{\text {tank }}$ on $\Phi$ is weak, as obvious from the same (19), a first estimate of $A_{\text {tank }}$ is obtained for $\Phi=0$, after which an approximation of $\Phi$, and subsequently a more accurate value of $A_{\text {tank }}$ are obtained

${ }^{4}$ If $C_{2}^{\prime \prime} \gg C_{2}^{\prime}$, the loop gain for common-mode signals is too low to sustain common-mode oscillations. If $C_{2}^{\prime}$ is large enough to allow common-mode oscillations, these can still be suppressed if the common-mode losses are high enough. The parasitic resistance of the metal interconnect between the common node of the two tank inductors and the power supply should in practical designs be sufficient to prevent common-mode oscillations. them through a center-tapped inductor. Both techniques may of course be employed in the same design, as in Fig. 1(b). The use of a center-tapped inductor alone results in a differential-mode inductance value of $L^{\prime}(1+k)$ and a common-mode inductance value of $L^{\prime}(1-k)$, where $k$ is the coupling factor between the two inductors of the center-tapped coil. Since $k$ is very high $(\approx 0.9)$, the quality factor of the common-mode inductance is too low to sustain steady-state oscillations, and only differential-mode oscillations survive. In this way, a floating $C_{2}^{\prime \prime}$ is not needed for the enforcement of differential-mode operations, which means that the oscillation frequency of the differential Colpitts can be aggressively increased by allowing most or all of $C_{2}$ in each of the two coupled oscillators to be made of parasitic $C_{\mathrm{gs}}$ and $C_{\mathrm{bs}}$ capacitances. A second, well-known advantage of using a center-tapped inductor is its higher quality factor, compared to two separate inductors (all other things being identical).

Assuming now that differential-mode oscillations are produced, it is clear that we can analyze the behavior of each of the two oscillators in Fig. 1(b) as if it were a standalone singleended oscillator, with an inductance value

$$
L=L^{\prime}(1+k)
$$

and a capacitance value

$$
C_{2}=C_{2}^{\prime}+2 C_{2}^{\prime \prime} \text {. }
$$

Equations (3)-(19) describe, therefore, both the single-ended and differential Colpitts oscillator, provided that all parameters are referred to the single-ended oscillator also when considering the differential case. As an example, $A_{\text {tank }}$ is the oscillation amplitude for each of the two phases, and $I_{B}$ is half of the total current consumption, when the differential case is considered.

\section{B. Phase Noise in the $1 / f^{2}$ Region}

In the following, we are going to derive an expression for the phase noise of the Colpitts oscillator, valid for both the single-ended and the differential topology. All equations will be referred to a single-ended oscillator output, the phase noise itself being independent of this choice [9].

Referring to a general oscillator, it has been shown [6], [10] that the presence of a resistance $R_{t}$ between one oscillator node and ground causes a phase noise $\mathcal{L}$, at the offset frequency $\Delta \omega$, given by

$$
\mathcal{L}(\Delta \omega)=10 \log \left(\frac{\Gamma_{R_{t}, \mathrm{rms}}^{2}}{q_{\max }^{2}} \cdot \frac{\overline{i_{R_{t}}^{2}} / \Delta f}{2 \Delta \omega^{2}}\right)
$$

where $q_{\max }$ is the maximum amount of dynamic charge loaded onto the capacitance in parallel to $R_{t}$ across an oscillation period, $\overline{i_{R_{t}}^{2}}$ is the power density of the stationary white noise current generated by $R_{t}$, given by the well-known expression

$$
\overline{i_{R_{t}}^{2}}=4 k_{B} T \frac{1}{R_{t}} \Delta f
$$

and $\Gamma_{R_{t}}$ is the ISF of such a noise source. In a previous work [9] we have shown that, in the special case of an $L C$-tank oscillator generating (almost) sinusoidal signals, $\Gamma_{R_{t}}$ is given by

$$
\Gamma_{R_{t}}(\phi)=\frac{\sin (\phi)}{N}
$$


where $N=1$ for a single-ended oscillator, and $N=2$ for a differential oscillator. ${ }^{5}$ Equation (24) assumes, without loss of generality, that the voltage at the node where $\overline{i_{R_{t}}^{2}}$ flows is of the form $-V_{p} \cos (\phi)$, i.e., it has the same phase as $V_{\text {tank }}(\phi)$. Thus, (24) can be directly used in its present form in the following analysis.

From (24), the square rms value of $\Gamma_{R_{t}}$ is

$$
\Gamma_{R_{t}, \mathrm{rms}}^{2}=\int_{-\pi}^{\pi} \Gamma_{R_{t}}^{2}(\phi) d \phi=\frac{1}{2 N^{2}} .
$$

When a cyclo-stationary noise source is considered, the same (22) valid for stationary noise sources can still be applied, provided that the ISF is replaced by an effective ISF $\Gamma_{\text {eff }}$ defined as [6]

$$
\Gamma_{\text {eff }}(\phi)=\Gamma(\phi) \cdot \alpha(\phi)
$$

where $\alpha(\phi)$ includes the dependence of the noise source power on $\phi$. Equation (26) will be used to study the effects of the MOS noise current $\overline{i_{\mathrm{ds}}^{2}}$, which is obviously cyclo-stationary. In order to find $\Gamma_{i_{\mathrm{ds}}}$, defined here as the ISF associated to $\overline{i_{\mathrm{ds}}^{2}}$, we turn to the definition of ISF as the excess phase generated by a current impulse injected into the same oscillator node where the noise current flows. It is convenient to model the drain noise current as two fully correlated grounded sources $\overline{i_{\mathrm{ds}, a}^{2}}$ and $\overline{i_{\mathrm{ds}, b}^{2}}$, each equal to $\overline{i_{\mathrm{ds}}^{2}}$, as in Fig. 3(b), where all circuit components except the two capacitors have been neglected, since impulsive currents flow entirely through the capacitive impedances (if present). Since $\overline{i_{\mathrm{ds}, a}^{2}}$ lies in parallel to $\overline{i_{R_{t}}^{2}}$, we can conclude immediately that $\overline{i_{\mathrm{ds}, a}^{2}}$ and $\overline{i_{R_{t}}^{2}}$ possess identical ISFs:

$$
\Gamma_{i_{\mathrm{ds}, a}}=\Gamma_{R_{t}} .
$$

Referring again to Fig. 3, voltage $\Delta V_{a, a}$, measured at node $a$ and generated by a current impulse of area $\Delta Q$ flowing into node $a$, is

$$
\Delta V_{a, a}=\frac{\Delta Q}{C}
$$

while voltage $\Delta V_{a, b}$, still measured at node $a$, and generated by a current impulse of the same area $\Delta Q$, flowing this time out of node $b$, is

$$
\begin{aligned}
\Delta V_{a, b} & =-\frac{\Delta Q}{C_{2}}=-\frac{\Delta V_{a, a} C}{C_{2}} \\
& =-\Delta V_{a, a} \frac{C_{1}}{C_{1}+C_{2}}=-n \Delta V_{a, a} .
\end{aligned}
$$

Considering that $\Delta V_{a, a}$ results in the ISF $\Gamma_{i_{\mathrm{ds}, a}}$, (29) yields the expression for $\Gamma_{i_{\mathrm{ds}, b}}$ as

$$
\Gamma_{i_{\mathrm{ds}, b}}=-n \Gamma_{i_{\mathrm{ds}, a}} .
$$

\footnotetext{
${ }^{5}$ Equation (24) was obtained under the assumption that the state equations for the oscillator were those provided by the voltage across the capacitor and the current through the inductor in each $L C$ tank [9]. In the case of a Colpitts oscillator there are, strictly speaking, three state equations for each phase, since the voltages across the two capacitors are in general independent of each other. However, the transistor loads $C_{2}$ lightly when the $Q$ of the Colpitts resonator is even moderately high, and neglecting such a loading effect, the series combination of $C_{1}$ and $C_{2}$ is equivalent to a single capacitor, with value given by (14). Therefore, (24) can be used in the study of Colpitts phase noise as well.
}

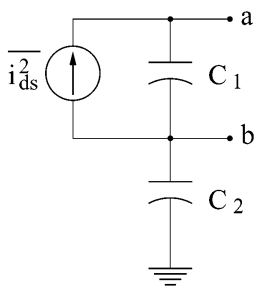

(a)

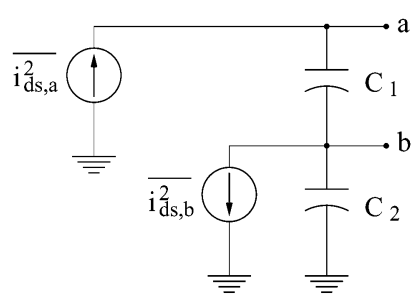

(b)
Fig. 3. Circuit for ISF calculations in a Colpitts oscillator.

In reality, (30) is only approximately true, since a charge $\Delta Q$ flowing into node $b$ does not charge $C_{1}$, as opposite to a charge $n \Delta Q$ flowing into node $a$. Using now (27) and (30), the ISF for the drain noise current becomes

$$
\begin{aligned}
\Gamma_{i_{\mathrm{ds}}} & =\Gamma_{i_{\mathrm{ds}, a}}+\Gamma_{i_{\mathrm{ds}, b}} \\
& =(1-n) \Gamma_{i_{\mathrm{ds}, a}}=(1-n) \Gamma_{R_{t}}=(1-n) \frac{\sin (\phi)}{N} .
\end{aligned}
$$

There remains to find the effective ISF associated to the drain noise current, which is easily done by noticing that (5) yields the transconductance $g_{m}$ as

$$
g_{m}=\beta A[\cos (\phi)-\cos (\Phi)]
$$

valid for $-\Phi \leq \phi \leq \Phi$. A commonly used expression for the noise current power of the MOS transistor is

$$
\begin{aligned}
\overline{i_{\mathrm{ds}}^{2}} & =4 k_{B} T \gamma g_{m} \Delta f=4 k_{B} T \gamma \beta A[\cos (\phi)-\cos (\Phi)] \Delta f \\
& \equiv \overline{i_{\mathrm{ds}}^{\prime 2}}[\cos (\phi)-\cos (\Phi)]
\end{aligned}
$$

where the simplifying assumption is made that $\overline{i_{\mathrm{ds}}^{2}}$ is proportional to $g_{m}$ through the factor $\gamma$, with $\gamma=2 / 3$ in the longchannel limit. Equation (33) contains the expression for $\alpha(\phi)$ in (26)

$$
\alpha(\phi)=\sqrt{\cos (\phi)-\cos (\Phi)} .
$$

From (26), (31), and (34), the effective ISF for the MOS noise current becomes

$$
\begin{aligned}
\Gamma_{i_{\mathrm{ds}}, \mathrm{eff}}(\phi) & =\Gamma_{i_{\mathrm{ds}}}(\phi) \cdot \alpha(\phi) \\
& =(1-n) \frac{\sin (\phi)}{N}[\cos (\phi)-\cos (\Phi)]^{\frac{1}{2}}
\end{aligned}
$$

with square rms value

$$
\begin{aligned}
\Gamma_{i_{\mathrm{d} s}, \mathrm{eff}, \mathrm{rms}}= & \frac{1}{2 \pi} \frac{(1-n)^{2}}{N^{2}} \int_{-\Phi}^{\Phi} \sin ^{2}(\phi)[\cos (\phi)-\cos (\Phi)] d \phi \\
= & \frac{1}{2 \pi} \frac{(1-n)^{2}}{N^{2}}\left[\frac{1}{3} \cos ^{2}(\Phi) \sin (\Phi)\right. \\
& \left.+\frac{2}{3} \sin (\Phi)-\Phi \cos (\Phi)\right] .
\end{aligned}
$$

Typical plots of $V_{\text {tank }}(\phi), I_{\mathrm{ds}}(\phi), \Gamma_{i_{\mathrm{ds}}}(\phi)$, and $\Gamma_{i_{\mathrm{ds}}, \mathrm{eff}}(\phi)$ are shown in Fig. 4.

The term in $\Phi$ in (36) is the same as that appearing in (9); thus, the expression of $\Gamma_{i_{\mathrm{ds}} \text {, eff,rms }}^{2}$ in terms of $I_{1}$ and $A_{s}$ is particularly simple

$$
\Gamma_{i_{\mathrm{ds}}, \mathrm{eff}, \mathrm{rms}}^{2}=\frac{(1-n)^{2}}{N^{2}} \frac{I_{1}}{2 \beta A_{s}^{2}} .
$$




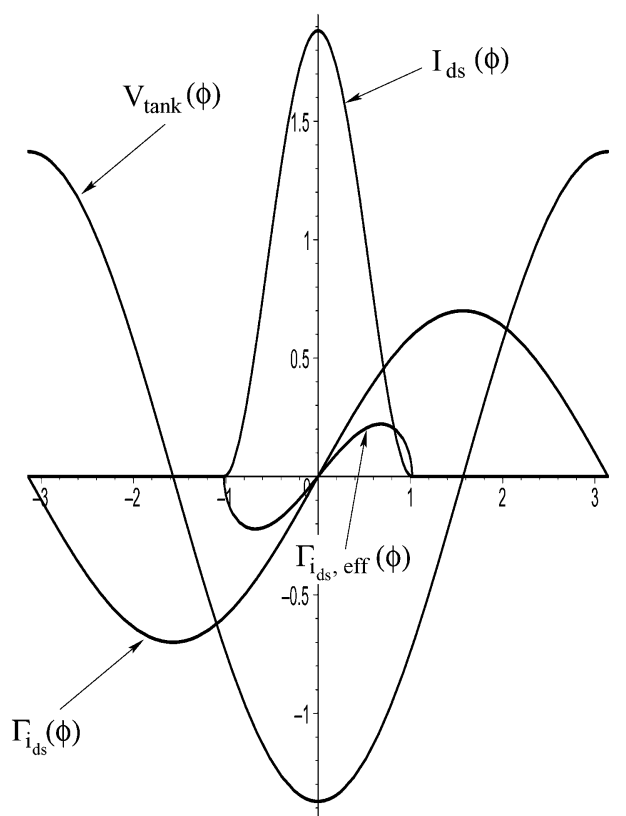

Fig. 4. Typical waveforms and ISFs in a Colpitts oscillator: $V_{\text {tank }}(\phi), I_{\mathrm{ds}}(\phi), \Gamma_{i_{\mathrm{ds}}}(\phi)$, and $\Gamma_{i_{\mathrm{ds}}, \text { eff }}(\phi)$, as produced by (17), (3), (31), and (35), respectively $\left(N=1\right.$, and $I_{\mathrm{ds}}(\phi)$ not to scale, for the sake of visibility).

We are finally able to write the phase noise expression for the Colpitts oscillator. Extending (22), $\mathcal{L}(\Delta \omega)$ is given by

$$
\begin{array}{r}
\mathcal{L}(\Delta \omega)=10 \log \left[N \cdot \frac { 1 } { 2 q _ { \mathrm { max } } ^ { 2 } \Delta \omega ^ { 2 } } \left(\Gamma_{i_{\mathrm{ds}}, \mathrm{eff}, \mathrm{rms}}^{\overline{i_{\mathrm{ds}}^{\prime 2}}} / \Delta f\right.\right. \\
\left.\left.+\Gamma_{R_{t}, \mathrm{rms}}^{2} \overline{i_{R_{t}}^{2}} / \Delta f\right)\right]
\end{array}
$$

where the factor $N$ accounts for the fact that in an $N$-phase oscillator there are $N$ identical uncorrelated noise sources for each noise mechanism, all contributing equally to phase noise [9]. Substituting the relevant equations into (38), we obtain

$$
\begin{aligned}
\mathcal{L}(\Delta \omega) & \\
= & 10 \log \left[\frac{4 k_{B} T}{2 N A_{\mathrm{tank}}^{2} C^{2} \Delta \omega^{2}}\left((1-n)^{2} \frac{I_{1}}{2 \beta A_{s}^{2}} \gamma \beta A_{s}+\frac{1}{2 R_{t}}\right)\right] \\
= & 10 \log \left[\frac{k_{B} T}{N A_{\mathrm{tank}}^{2} C^{2} \Delta \omega^{2}}\left((1-n) \gamma \frac{1}{n R_{t}}+\frac{1}{R_{t}}\right)\right] \\
= & 10 \log \left[\frac{k_{B} T}{4 N I_{B}^{2} R_{t}^{3} C^{2}\left(1-\Phi^{2} / 14\right)^{2} \Delta \omega^{2}}\right. \\
& \left.\times\left(\frac{\gamma}{n(1-n)}+\frac{1}{(1-n)^{2}}\right)\right]
\end{aligned}
$$

which, to repeat, is valid for both the single-ended Colpitts $(N=1)$, and the differential Colpitts $(N=2)$. Perhaps surprisingly, the dependence of $\mathcal{L}$ on $\Phi$ is very weak and can be neglected in a first-order approximation; otherwise, substituting $\Phi$ with (4) yields excellent results even for very large values of the conduction angle. Remarkably, (39) shows that the value of $n$ resulting in minimum phase noise depends on $\gamma$ and nothing else. Straightforward derivation of $\mathcal{L}$ yields the optimal $n$ as

$$
\begin{aligned}
n_{\mathrm{opt}} & =\frac{3 \gamma-\sqrt{\gamma^{2}+8 \gamma}}{4(\gamma-1)} \\
& =\frac{2}{3+\sqrt{1+8 / \gamma}} \\
& = \begin{cases}0.30, & \text { for } \gamma=2 / 3 \\
1 / 3, & \text { for } \gamma=1 \\
0.36, & \text { for } \gamma=1.5\end{cases}
\end{aligned}
$$

A normalized plot of (39) versus $n$ for $\gamma=2 / 3$ is shown in Fig. 5, from which it is clear that the phase-noise minimum is a relatively flat one. [For the slightly different Colpitts oscillator in [7], it can be shown that

$$
\begin{aligned}
& \mathcal{L}(\Delta \omega) \\
& =10 \log \left[\frac{k_{B} T}{4 N I_{B}^{2} R_{t}^{3} C^{2}\left(1-\Phi^{2} / 14\right)^{2} \Delta \omega^{2}}\left(\frac{\gamma}{n(1-n)}+\frac{1}{n^{2}}\right)\right] .
\end{aligned}
$$

Comparing (39) and (41), it is clear that both Colpitts designs are capable of exactly the same minimum value of phase noise, but for different values of $n$. This is obvious once it is realized that (41) is obtained from (39) with the substitution $n \rightarrow 1-n$, which is equivalent to interchanging the values of $\mathrm{C}_{1}$ and $\mathrm{C}_{2}$. As an example, if $\gamma$ is unity, minimum phase noise is now achieved with $n=2 / 3$.]

For the sake of completeness, we extend (39) to include the effects of a stationary noisy bias source with equivalent noise admittance $g_{m T}$. The contribution of this noise source is readily calculated, once it is noticed that its ISF is equal to $\Gamma_{i_{\mathrm{ds}, b}}$. The new phase-noise expression becomes

$$
\begin{aligned}
\mathcal{L}(\Delta \omega)= & 10 \log \left[\frac{k_{B} T}{4 N I_{B}^{2} R_{t}^{3} C^{2}\left(1-\Phi^{2} / 14\right)^{2} \Delta \omega^{2}}\right. \\
& \left.\times\left(\frac{\gamma}{n(1-n)}+\frac{1}{(1-n)^{2}}+\frac{n^{2} R_{t} g_{m T}}{(1-n)^{2}}\right)\right]
\end{aligned}
$$

In a well-designed oscillator, the contribution from the bias noise is negligible; otherwise, (42) can be numerically minimized versus $n$.

Finally, it is easy to show that (42) does not change, even if an arbitrarily large part of the resonator capacitance is directly connected between output and ground (maintaining oscillation frequency and resonator- $Q$ constant, of course). This remark is of interest because such a capacitance is unavoidable in practice, due to the presence of the parasitic substrate capacitance of the integrated inductor, and to the drain diode of the MOS transistor.

1) Theory Versus SpectreRF Simulations: The analog simulator spectreRF calculates the phase noise of any oscillator as the ratio of the phase-noise-generating noise contributions from the various components, to the power of the output signal. In the case of a differential oscillator with sinusoidal output waveforms, the spectreRF expression for $\mathcal{L}(\Delta \omega)$ is

$$
\mathcal{L}(\Delta \omega)=10 \log \left(2 \frac{N_{\mathcal{L}_{R_{t}}}+N_{\mathcal{L}_{i_{\mathrm{ds}}}}}{A_{p}^{2} / 2}\right)
$$




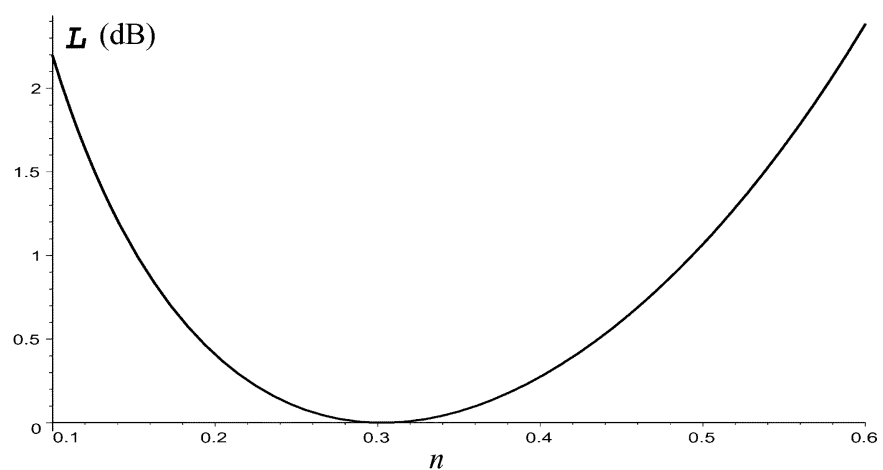

Fig. 5. Normalized Colpitts phase noise versus $n$ with $\gamma=2 / 3$, as from (39).

where $N_{\mathcal{L}_{R_{t}}}$ and $N_{\mathcal{L}_{i_{\mathrm{ds}}}}$ are the phase-noise-generating noise contributions from one parallel tank resistance and one MOS transistor, the factor 2 at the numerator accounts for the presence of two noise sources of the same kind in a differential oscillator (noise contributions from any bias sources are neglected for simplicity), and $A_{p}$ is the peak value of the sinusoidal oscillation. As in the preceding analysis, we assume that both noise contributions and oscillation waveforms are referred to a single-ended output of each oscillator.

Table I shows results of phase-noise simulations performed with spectreRF, for the $2.5-\mathrm{GHz}$ differential Colpitts oscillator of Fig. 1(b), having a resonator- $Q$ of 12, equivalent inductance value $L^{\prime}(1+k)=1.3 \mathrm{nH}$, MOS transistors of dimensions $W / L=500 \mu \mathrm{m} / 0.35 \mu \mathrm{m}$, and a bias current of $2 \times 4 \mathrm{~mA}$. In order to check the validity of the equations previously derived, simulations were run with both real and "ideal" BSIM3v.3 MOS simulation models (where we call "ideal" the BSIM3v.3 model stripped of the mobility reduction effects caused by the normal and transversal channel electric fields). It can be appreciated that calculations and "ideal" simulations yield (almost) identical data for all values of $n$. These results, we believe, show the power of the ISF theory as an analysis tool for oscillator phase noise, whenever more than numerical results are desired. This issue will be expanded upon in the next section.

\section{TIME-INVARIANT VERSUS TIME-VARIANT PHASE-NOISE ANALYSIS}

It is interesting to compare the phase noise expression in (39) with what would be obtained with a time-invariant analysis. As clear from (25), the ISF theory leads to a conversion factor of $1 / 2$, or a fraction of it through $N^{2}$ in general, between total noise and phase-noise-generating noise, under the assumption that the noise source is stationary and the oscillation waveform is sinusoidal. As a matter of fact, such a factor $1 / 2$ is often recovered employing other more or less rigorous methods as well, and it is perhaps difficult to regard it as a compelling proof of the need of a time-variant approach.

More revealing is an analysis of the cyclo-stationary noise generated by the MOS transistor(s), since in this case the fundamental role played by a non-constant ISF becomes obvious and its importance unquestionable, at least at a theoretical level. Turning therefore without further delay to the relevant equations, if we choose to neglect the different impact that noise has
TABLE I

Calculated and Simulated Noise CONTRIButions (IN $10^{-15} \cdot \mathrm{V}_{\mathrm{rms}}^{2} / \mathrm{Hz}$ ) AND PHASE NoISE (IN $-\mathrm{dBc} / \mathrm{Hz}$ ) AT 1-MHz OFFSET FREQUENCY, FOR THE 2.5-GHz DifFERENTIAL COLPITTS OSCILlator OF FIG. 1(b)

\begin{tabular}{llllllll}
\hline \hline $\mathrm{n}$ & 0.097 & 0.194 & 0.289 & 0.385 & 0.481 & 0.577 & 0.676 \\
\hline$N_{\mathcal{L}_{R_{t}}}$ (calc.) & 5.5 & 5.5 & 5.5 & 5.4 & 5.4 & 5.3 & 5.2 \\
$N_{\mathcal{L}_{R_{t}}}$ (sim.) & 5.5 & 5.5 & 5.5 & 5.3 & 5.3 & 5.3 & 5.7 \\
$N_{\mathcal{L}_{i_{d s}}}$ (calc.) & 34.3 & 15.3 & 9.0 & 5.8 & 3.9 & 2.6 & 1.6 \\
$N_{\mathcal{L}_{i_{d s}}}$ (sim. ideal) & 33.2 & 15.3 & 9.1 & 5.9 & 4.0 & 2.7 & 1.8 \\
$N_{\mathcal{L}_{i_{d s}}}$ (sim. real) & 45.2 & 21.0 & 12.1 & 7.7 & 5.1 & 3.5 & 2.3 \\
\hline $\mathcal{L}$ (calc.) & 130.5 & 133.5 & 134.3 & 134.2 & 133.6 & 132.4 & 130.6 \\
$\mathcal{L}$ (sim. ideal) & 129.9 & 133.3 & 134.1 & 134.0 & 133.4 & 132.1 & 130.0 \\
\hline \hline
\end{tabular}

on phase noise at different instants across the signal period, the dependence of (31) on $\phi$ has to go. Therefore, $\sin (\phi)$ in (31) should be replaced by a constant. If we want to obtain the same aforementioned conversion factor of $1 / 2$ for cyclo-stationary sources as well (although there is of course no justification for this choice), this constant should be $1 / \sqrt{2}$, or, which is the same, $\sin ^{2}(\phi)$ in (36) should be replaced by the factor $1 / 2$. Equation (36) becomes now proportional to the mean of $\alpha(\phi)$ over one period; calling $\Gamma_{\text {wrong }}^{2}$ this new value for (36), we obtain

$$
\begin{aligned}
\Gamma_{\text {wrong }}^{2} & =\frac{1}{2} \cdot \frac{1}{2 \pi} \frac{(1-n)^{2}}{N^{2}} \int_{-\Phi}^{\Phi} \alpha(\phi) d \phi \\
& =\frac{1}{2 \pi} \frac{(1-n)^{2}}{N^{2}}[\sin (\Phi)-\Phi \cos (\Phi)]
\end{aligned}
$$

From (36) and (44), it is easy to calculate the error we make if a time-variant phase noise theory is not adopted; defining $\Delta N_{\mathcal{L}_{i \mathrm{ds}}}$ as the excess noise factor introduced by (44), we obtain

$$
\begin{aligned}
\Delta N_{\mathcal{L}_{i \mathrm{ds}}} & \equiv \frac{\Gamma_{\mathrm{wrong}}^{2}}{\Gamma_{i_{\mathrm{ds}}, \mathrm{eff}, \mathrm{rms}}^{2}} \\
& =\frac{\sin (\Phi)-\Phi \cos (\Phi)}{\frac{1}{3} \cos ^{2}(\Phi) \sin (\Phi)+\frac{2}{3} \sin (\Phi)-\Phi \cos (\Phi)} \\
& \approx \frac{5}{2 \Phi^{2}}+\frac{17}{42}
\end{aligned}
$$

where it is clear that $\Delta N_{\mathcal{L}_{i \mathrm{ds}}}$ can be very large for small conduction angles. A plot of the excess phase noise given by (45) is shown in Fig. 6 , for $\Phi$ between 0.6 and 1.4; as an example, even for a moderate $\Phi$ of one radian, $\Delta N_{\mathcal{L}_{i \mathrm{ds}}}$ becomes approximately 2.93 , which corresponds to an overestimation as large as $4.8 \mathrm{~dB}$ for the phase noise caused by the transistor (the overestimation of the total phase noise is $\sim 3.2 \mathrm{~dB}$ ). Equation (45), together with the simulation results of Section II-B1), shows the importance of adopting a time-variant approach in the study of phase noise.

\section{Phase Noise AnAlysis In $L C$-TAnK Oscillators}

The $1 / f^{2}$ phase noise of the $L C$-tank oscillator in Fig. 2 will be treated in this section. In particular, we will derive an expression for the phase noise generated by the noise in the transistor channels, thereby showing that such a phase noise and the phase noise generated by the tank resistors are in a ratio $\gamma: 1$, and this 
for a very wide range of working conditions of the oscillator; ${ }^{6}$ further, a new, general equation for the phase noise generated by the tail current noise will be obtained as well. We will here restrict our analysis to a simplified model of the $L C$-tank oscillator, where the tank capacitances are much larger than all parasitic capacitances in the circuit.

With arbitrary initial phase for the sinusoids at the tank outputs, we can write

$$
V_{\mathrm{tank},+}(\phi)=A_{\mathrm{tank}} \sin (\phi), \quad V_{\mathrm{tank},-}(\phi)=-A_{\mathrm{tank}} \sin (\phi) .
$$

Calling $I_{1}$ and $I_{2}$ the drain current of $M_{1}$ and $M_{2}$, we obtain

$$
\begin{aligned}
& I_{1}(\phi)=\frac{\beta}{2}\left(A_{\text {tank }} \sin (\phi)+V_{s}(\phi)\right)^{2} \\
& I_{2}(\phi)=\frac{\beta}{2}\left(-A_{\text {tank }} \sin (\phi)+V_{s}(\phi)\right)^{2}
\end{aligned}
$$

where $V_{s}(\phi)$ must be determined. Assuming that the parasitic tail capacitance $C_{T}$ is small, we can neglect the current through it, which leads to the following relation:

$$
I_{1}(\phi)+I_{2}(\phi)=2 I_{B}
$$

Straightforward algebra yields

$$
V_{s}(\phi)=\sqrt{\frac{2 I_{B}}{\beta}-A_{\mathrm{tank}}^{2} \sin ^{2}(\phi)} .
$$

Substituting (50) in (47) or (48), we obtain the limit angle $\Phi$ as

$$
\sin ^{2}(\Phi)=\frac{I_{B}}{\beta A_{\mathrm{tank}}^{2}} \rightarrow \Phi=\arcsin \sqrt{\frac{I_{B}}{\beta A_{\mathrm{tank}}^{2}}} .
$$

$\Phi$ divides the operation mode of the differential pair into two regions: for $-\Phi<\phi<\Phi$ or $\pi-\Phi<\phi<\pi+\Phi$, both transistors are working in saturation; ${ }^{7}$ otherwise, one of the transistors is turned off. Rewriting $V_{s}(\phi)$ as

$$
V_{s}(\phi)=A_{\operatorname{tank}} \sqrt{2 \sin ^{2}(\Phi)-\sin ^{2}(\phi)}
$$

(47), (48), and (52) yield the transistor transconductances as

$$
\begin{aligned}
& g_{m 1}(\phi)=\beta A_{\mathrm{tank}}\left(\sin (\phi)+\sqrt{2 \sin ^{2}(\Phi)-\sin ^{2}(\phi)}\right) \\
& g_{m 2}(\phi)=\beta A_{\mathrm{tank}}\left(-\sin (\phi)+\sqrt{2 \sin ^{2}(\Phi)-\sin ^{2}(\phi)}\right) .
\end{aligned}
$$

Once again, we will relate the ISF associated to the transistor noise, to the ISF associated to the tank noise; because of symmetry, it is enough to study $\Gamma_{i_{\mathrm{ds} 1}}$, defined as the ISF relative to $M_{1}$. Given (46), the ISFs at nodes tank+ and tank-, respectively, are given by [9]

$$
\Gamma_{R_{t},+}(\phi)=\frac{\cos (\phi)}{N}, \quad \Gamma_{R_{t},-}(\phi)=-\frac{\cos (\phi)}{N} .
$$

To repeat, $\Gamma_{R_{t},+}$ is associated to the excess phase generated by a current impulse of area $\Delta Q$, which flows between ground and

\footnotetext{
${ }^{6}$ This result has already been stated, without a detailed proof and under the limiting assumption of square-wave current waveforms, in [11].

${ }^{7}$ This is true if $\Phi<\left(V_{\mathrm{th}}\right) /\left(2 A_{\mathrm{tank}}\right)$, where $V_{\mathrm{th}}$ is the threshold voltage of the transistors. This is not difficult to achieve in practice.
}

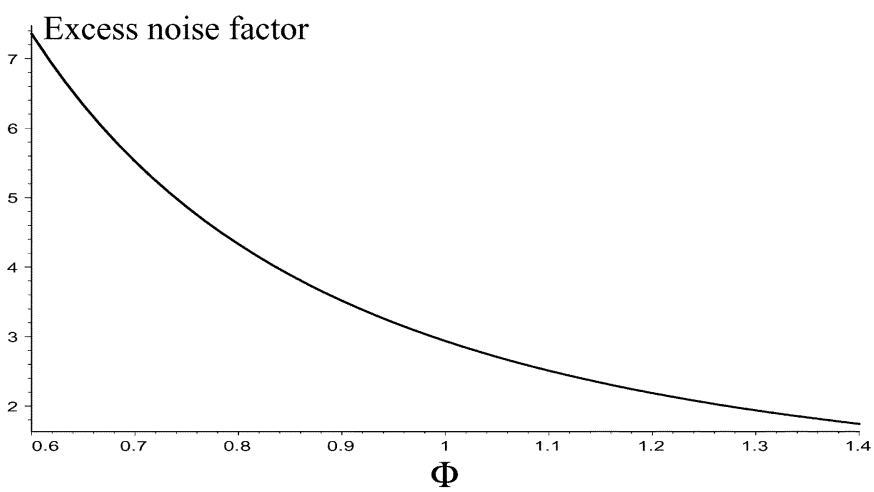

Fig. 6. Excess noise factor in Colpitts phase-noise analysis, as from (45).

tank + , charging the tank capacitance ${ }^{8}$ to $\Delta V$. To find $\Gamma_{i_{\mathrm{ds} 1}}$, we must send the same current impulse between drain and source of $M_{1}$, as in Fig. 7; $C_{1}$ is again charged to $\Delta V$, while $C_{T}$ is charged by $-\Delta Q$ to some voltage $-\Delta V_{T}$. We distinguish now two cases, according to the values taken by $\phi$. If $\Phi<\phi<$ $\pi-\Phi, M_{2}$ is off, while $M_{1}$ conducts the whole tail current. If $C_{T}$ is small, so that the time constant $C_{T} / g_{m 1}$ is much smaller than the period of the oscillations, the charge $-\Delta Q$ is moved from $C_{T}$ to $C_{1}$ approximately at the same instant when $C_{1}$ is charged to $\Delta V$ by $\Delta Q$, as shown in Fig. 7(a). The net voltage variation on $C_{1}$ is, therefore, zero, and so is $\Gamma_{i_{\mathrm{ds} 1}}$. This result is not unexpected, since it is only another form of the well-known noise rejection mechanism in a cascode transistor with large source degeneration. It may be useful to point out that $\Gamma_{i_{\mathrm{ds} 1}}$ is still zero, even if $M_{1}$ enters the triode/linear region for some angle inside $\Phi<\phi<\pi-\Phi$.

The second case we have to take into account is for $-\Phi<$ $\phi<\Phi$ and $\pi-\Phi<\phi<\pi+\Phi$, when both transistors are working in saturation. Reasoning again on $\Delta Q$, the situation is the one depicted in Fig. 7(b). The current impulse generates now two voltages, $\Delta V_{1}$ on $C_{1}$ and $\Delta V_{2}$ on $C_{2}$, with values ${ }^{9}$

$$
\begin{aligned}
& \Delta V_{1}=\Delta V \frac{g_{m 2}}{g_{m 1}+g_{m 2}} \\
& \Delta V_{2}=-\Delta V \frac{g_{m 2}}{g_{m 1}+g_{m 2}} .
\end{aligned}
$$

Considering that $\Delta V_{1}$ and $\Delta V_{2}$ are obviously fully correlated, and making use of (55), the excess phase caused by $\Delta V_{1}$ and $\Delta V_{2}$ is equivalent to the excess phase caused by a single $\Delta V_{1}^{\prime}$ on $C_{1}$, with value

$$
\Delta V_{1}^{\prime}=\Delta V_{1}-\Delta V_{2}=\Delta V \frac{2 g_{m 2}}{g_{m 1}+g_{m 2}} .
$$

Since $\Delta V$ generates $\Gamma_{R_{t},+},(58)$ allows us to write $\Gamma_{i_{\mathrm{ds} 1}}$ as

$$
\Gamma_{i_{\mathrm{ds} 1}}(\phi)=\frac{\cos (\phi)}{N} \frac{2 g_{m 2}(\phi)}{g_{m 1}(\phi)+g_{m 2}(\phi)} .
$$

Finally, the value of $\Gamma_{i_{\mathrm{ds} 1}}$ for $\pi+\Phi<\phi<2 \pi-\Phi$ is obviously uninteresting, since in this region $M_{1}$ is off and does not contribute any noise.

\footnotetext{
${ }^{8}$ In the following calculations, we will call $C_{1}\left(C_{2}\right)$ the capacitance at node tank+ (tank-), with $C_{1}=C_{2}=C$.

${ }^{9}$ If $C_{T} \ll C$, then $\Delta V_{T} \gg \Delta V$, and $g_{m} V_{\mathrm{gs}} \approx-g_{m} V_{s}=-g_{m} \Delta V_{T}$ for both transistors, from which (56)-(57) follow.
} 


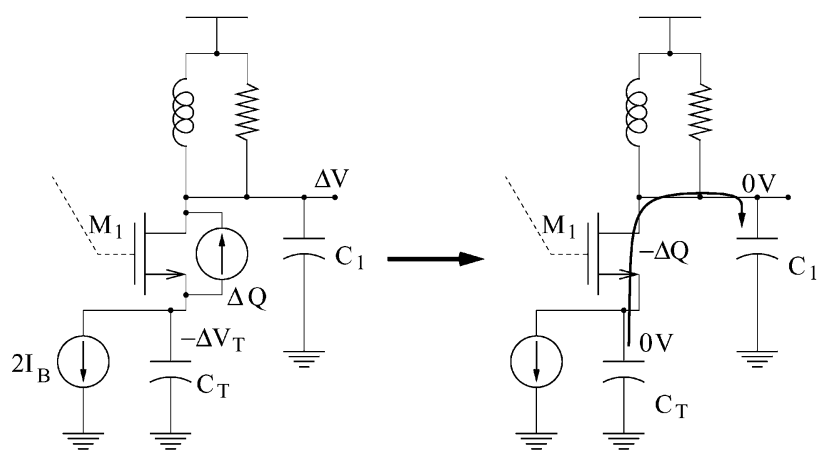

(a)
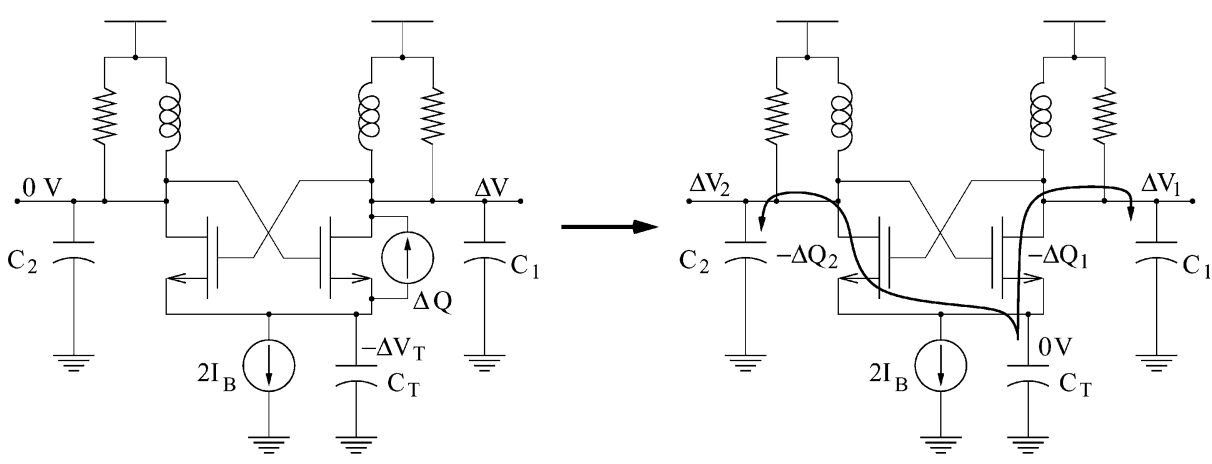

(b)

Fig. 7. ISF derivation in the $L C$-tank oscillator, when: (a) only $M_{1}$ is in the on-state and (b) both transistors are in the on-state.

To find the effective ISF $\Gamma_{i_{\mathrm{ds} 1}}$,eff, we use again (26), where $\alpha(\phi)$ is obtained from (53) as

$$
\alpha(\phi)=\sqrt{\sin (\phi)+\sqrt{2 \sin ^{2}(\Phi)-\sin ^{2}(\phi)}} .
$$

After simplifying the product $\Gamma_{i_{\mathrm{ds} 1}}^{2}(\phi) \alpha^{2}(\phi), \Gamma_{i_{\mathrm{ds} 1}, \text { eff,rms }}^{2}$ is written as

$$
\begin{aligned}
& \Gamma_{i_{\mathrm{ds} 1}, \mathrm{eff}, \mathrm{rms}}^{2} \\
& =\frac{1}{2 \pi} \int_{-\Phi}^{2 \pi-\Phi} \Gamma_{i_{\mathrm{ds} 1}}^{2}(\phi) \alpha^{2}(\phi) d \phi \\
& =\frac{2}{\pi N^{2}} \int_{-\Phi}^{\Phi} \cos ^{2}(\phi) \\
& \quad \cdot \frac{\left(-\sin (\phi)+\sqrt{2 \sin ^{2}(\Phi)-\sin ^{2}(\phi)}\right)\left(\sin ^{2}(\Phi)-\sin ^{2}(\phi)\right)}{2 \sin ^{2}(\Phi)-\sin ^{2}(\phi)} d \phi
\end{aligned}
$$

where we have made use of the fact that the integral over $-\Phi<$ $\phi<\Phi$ is the same as over $\pi-\Phi<\phi<\pi+\Phi$. Making the change of integration variable $x=\sin (\phi)$, and setting $a=$ $\sin (\Phi),(61)$ can be integrated as

$$
\begin{aligned}
& \Gamma_{i_{\mathrm{ds} 1}, \mathrm{eff}, \mathrm{rms}}^{2} \\
& \quad=\frac{2}{\pi N^{2}} \int_{-a}^{a} \sqrt{1-x^{2}} \frac{\left(-x+\sqrt{2 a^{2}-x^{2}}\right)\left(a^{2}-x^{2}\right)}{2 a^{2}-x^{2}} d x \\
& \quad=\frac{2}{\pi N^{2}}\left[a^{2}+\left(-\frac{1}{2}+\frac{\pi}{8}\right) a^{4}+O\left(a^{6}\right)\right]
\end{aligned}
$$

which is excellently approximated by

$$
\Gamma_{i_{\mathrm{ds} 1}, \mathrm{eff}, \mathrm{rms}}^{2} \approx \frac{2}{\pi N^{2}} a^{2}=\frac{2}{\pi N^{2}} \sin ^{2}(\Phi)=\frac{2}{\pi N^{2}} \frac{I_{B}}{\beta A_{\mathrm{tank}}^{2}}
$$

The same approximated result would have been achieved if the factor $\cos (\phi)$ in $\Gamma_{i_{\mathrm{ds} 1}}$ had been replaced with unity, since $\cos ^{2}(\phi)$ in (61) is very close to unity for small values of $\Phi$, a condition which is easily satisfied in practice. Even for $\Phi$ as large as $\pi / 2$, setting $\cos (\phi)=1$ would only cause a modest $37 \%$ phase-noise overestimation. These facts lead to the following nontrivial consideration: apart from the factor $N$ in the ISFs, for small/moderate values of $\Phi$ all noise from $M_{1}$ is converted into phase noise, not only half of it, as is the case for the noise from the tank resistance.

Noticing the obvious fact that $\Gamma_{R_{t}, \mathrm{rms}}^{2}$ is still given by (25), and remembering that

$$
A_{\mathrm{tank}} \approx \frac{2}{\pi}\left(2 I_{B}\right) R_{t}
$$

when $\Phi$ is small, we use (38) to obtain the total phase-noise expression for the $L C$-tank oscillator:

$$
\begin{aligned}
\mathcal{L}(\Delta \omega)= & 10 \log \left[N \frac { 4 k _ { B } T } { 2 A _ { \mathrm { tank } } ^ { 2 } C ^ { 2 } \Delta \omega ^ { 2 } } \left(\frac{2}{\pi N^{2}} \frac{I_{B}}{\beta A_{\mathrm{tank}}^{2}}\right.\right. \\
& \left.\left.\cdot \gamma \beta A_{\mathrm{tank}}+\frac{1}{2 N^{2} R_{t}}\right)\right] \\
= & 10 \log \left[\frac{k_{B} T}{N A_{\mathrm{tank}}^{2} C^{2} \Delta \omega^{2} R_{t}}(\gamma+1)\right] .
\end{aligned}
$$


The above equation proves the ratio $\gamma: 1$ between the phase noise generated by transistors and tank resistors, respectively, a result stated at the beginning of this section.

As a matter of fact, we can prove a considerably stronger result: the very simple expression in (65) for the phase noise contributions from the transistors is not dependent on the particular value of $A_{\text {tank }}$ given by (64). In fact, if the dependency of $A_{\mathrm{tank}}$ on $\Phi$ is taken into account, together with the full expression of $\Gamma_{i_{\mathrm{ds} 1}, \text { eff,rms }}^{2}(\Phi)$, it is possible to show (with Maple's invaluable help) that the $\gamma: 1$ ratio is constant over the whole range $0<\Phi<\pi / 2$ (under the assumption that transistors never leave saturation for $-\Phi<\phi<\Phi$ ). This remarkable result, numerically verified for several values of $\Phi$, is another proof of the power of the ISF theory.

\section{A. Tail Current Noise}

Following the same approach as in the previous ISF calculations, the ISF $\Gamma_{\text {tail }}(\phi)$ of the tail noise current is found to be as given in (66), shown at the bottom of the page. It is easy to see that $\Gamma_{\text {tail }}(\phi)$ has double the frequency of $\Gamma_{R_{t}}(\phi)$, as expected; Fig. 8 shows $\Gamma_{\text {tail }}(\phi)$ for $\Phi=10^{\circ}$. $\Gamma_{\text {tail,rms }}^{2}$, found by numerically integrating $\Gamma_{\text {tail }}(\phi)$ over its period, is

$$
\Gamma_{\text {tail,rms }}^{2}(\Phi)=\frac{1}{2 N^{2}} \eta(\Phi)
$$

where $\eta(\Phi)$ is plotted in Fig. 9. In particular, $\eta(0)=1$ and $\eta(\pi / 2) \approx 0.17$.

The complete phase-noise expression becomes

$$
\begin{aligned}
& \mathcal{L}(\Delta \omega) \\
& \quad=10 \log \left[\frac{k_{B} T}{N A_{\text {tank }}^{2} C^{2} \Delta \omega^{2} R_{t}}\left(\gamma+1+\frac{\eta}{N} \gamma g_{m T} R_{t}\right)\right] .
\end{aligned}
$$

Equation (68) predicts that the phase noise generated by the tail current noise can be decreased by letting the differential pair switch more softly; this is easily confirmed quantitatively by spectreRF simulations.

\section{Comparison Between the Differential ColpitTs OSCILLATOR AND THE DIFFERENTIAL $L C$-TANK OSCILLATOR}

Let us assume that a differential Colpitts and an $L C$-tank oscillator are designed for the same oscillation frequency, use the same power consumption, are built around the same inductors

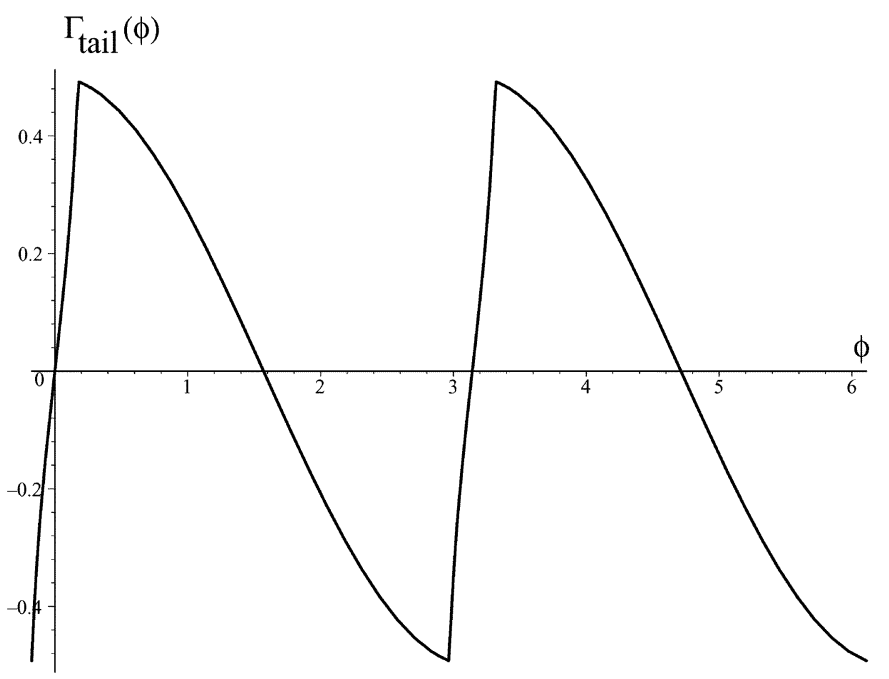

Fig. 8. ISF of the tail noise current for $\boldsymbol{\Phi}=10^{\circ}$.

$L$, suffer from the same losses, and generate sinusoidal oscillations. In the following, we are going to compare their phase noises as given by (43); specifically, we are going to compare their $N_{\mathcal{L}_{R_{t}}}, A_{p}$, and $N_{\mathcal{L}_{i_{\mathrm{ds}}}}$.

Starting with the signal amplitudes, the peak voltage of the sinusoidal oscillation for Colpitts and $L C$-tank oscillators are given by (19) and (64), respectively. The ratio of the two voltages is then

$$
\frac{A_{p, \mathrm{LC}}}{A_{p, \text { Colpitts }}}=\frac{2}{\pi(1-n)\left(1-\frac{\Phi_{\text {Colpitts }}^{2}}{14}\right)}
$$

which evaluates to 0.98 if we assume $n=0.30$ (optimal $n$ if $\gamma=2 / 3$, according to (40)) and a reasonable value of one radian for $\Phi_{\text {Colpitts. }}$. We can therefore assume that the two oscillators generate signals with the same amplitude.

Turning now to $N_{\mathcal{L}_{R_{t}}}$, it is necessary to check that the following equation applies to both oscillators:

$$
N_{\mathcal{L}_{R_{t}}}(\Delta \omega)=\frac{\Gamma_{\mathrm{rms}}^{2} \overline{i_{n}^{2}} / \Delta f}{4 C^{2} \Delta \omega^{2}}=\frac{k_{B} T}{2 N^{2} C^{2} R_{t} \Delta \omega^{2}} .
$$

Equation (70) shows that $N_{\mathcal{L}_{R_{t}}}$ is actually independent of the specific oscillator under consideration, a result already obtained in [9] (however, it is worth repeating that this is based on the assumption of sinusoidal waveforms).

$$
\Gamma_{\text {tail }}(\phi)= \begin{cases}\frac{g_{m 1}-g_{m 2}}{g_{m 1}+g_{m 2}} \Gamma_{R_{t},+}=\frac{\sin (\phi)}{\sqrt{2 \sin ^{2}(\Phi)-\sin ^{2}(\phi)}} \frac{\cos (\phi)}{N}, & \text { for }-\Phi<\phi<\Phi \\ \Gamma_{R_{t},+}=\frac{\cos (\phi)}{N}, & \text { for } \Phi<\phi<\pi-\Phi \\ \frac{g_{m 2}-g_{m 1}}{g_{m 1}+g_{m 2}} \Gamma_{R_{t},-}=\frac{\sin (\phi)}{\sqrt{2 \sin ^{2}(\Phi)-\sin ^{2}(\phi)} \frac{\cos (\phi)}{N},} & \text { for } \pi-\Phi<\phi<\pi+\Phi \\ \Gamma_{R_{t},-}=-\frac{\cos (\phi)}{N}, & \text { for } \pi+\Phi<\phi<2 \pi-\Phi\end{cases}
$$




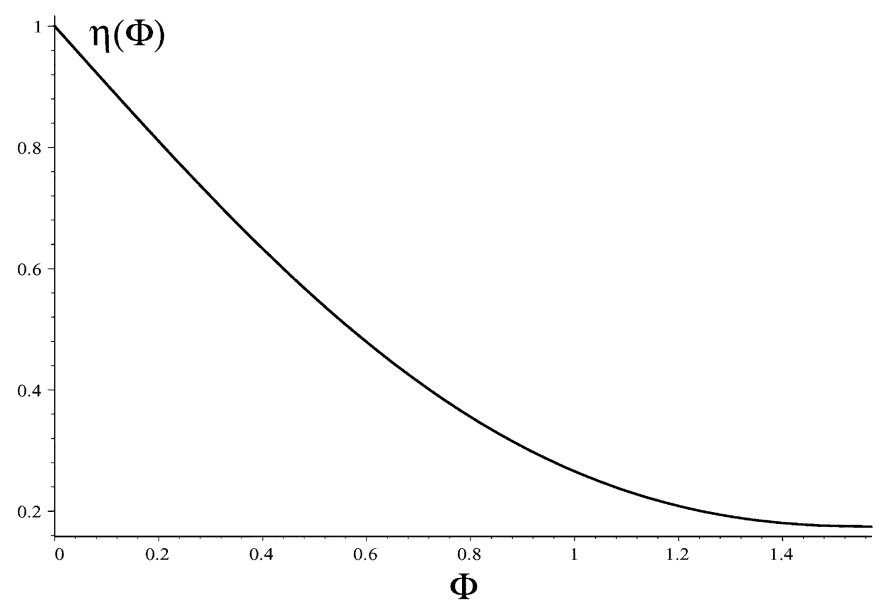

Fig. 9. Tail current phase-noise coefficient $\eta(\boldsymbol{\Phi})$ versus $\boldsymbol{\Phi}$.

There remains to examine $N_{\mathcal{L}_{i_{\mathrm{ds}}}}$. From (39) and (65), we obtain

$$
\frac{N_{\mathcal{L}_{i_{\mathrm{ds}}}, \text { Colpitts }}}{N_{\mathcal{L}_{R_{t}}, \mathrm{LC}-\text { tank }}}=\frac{\gamma_{\text {Colpitts }}}{\gamma_{\mathrm{LC}-\text { tank }}} \frac{(1-n)}{n}
$$

where $n$ is given by $n_{\text {opt }}$ in (40). If $\gamma_{\text {Colpitts }} \approx \gamma_{\mathrm{LC}-\text { tank }},(71)$ shows that the MOS transistors in the differential Colpitts oscillator contribute considerably more noise than in the $L C$-tank oscillator. As an example, $N_{\mathcal{L}_{i_{\mathrm{ds}}}}$, Colpitts is twice as large as $N_{\mathcal{L}_{R_{t}}, \mathrm{LC}-\text { tank }}$ when $\gamma$ is unity, resulting in a $\sim 2 \mathrm{~dB}$ higher overall phase noise for the Colpitts oscillator (neglecting the impact of tail current noise in both cases).

We can therefore conclude that the $L C$-tank oscillator displays a lower phase noise than the Colpitts oscillator in the $1 / f^{2}$ region, since it has the same $A_{p}$ and $N_{\mathcal{L}_{R_{t}}}$, but a lower $N_{\mathcal{L}_{i_{\mathrm{ds}}}}$.

In reality, things are even worse for Colpitts, for two reasons. First, subthreshold conduction and charge-carrier velocity saturation in MOS transistors tend to increase the value of $\Phi_{\text {Colpitts }}$, compared to when the ideal square-law $I_{\mathrm{ds}}-V_{\mathrm{gs}}$ relation is adopted; the oscillation amplitude then decreases, according to (19), leading to an increase in phase noise. Secondly, the large overdrive and drain-source voltages needed to create sharp current pulses have an adverse impact on the transistor transconductance as well, which can be roughly accounted for by increasing $\gamma_{\text {Colpitts }}$, resulting in a higher $N_{\mathcal{L}_{i_{\mathrm{ds}}}}$. This effect is already visible in the simulations of the $0.35 \mu \mathrm{m}$ CMOS technology and MOS models available to us, as shown in Table I: the real-MOS-model $N_{\mathcal{L}_{i_{\mathrm{ds}}}}$ is $30 \%$ or more larger than the idealMOS-model $N_{\mathcal{L}_{i_{\mathrm{ds}}}}$.

The $L C$-tank oscillator, on the other hand, is not as sensitive to these second-order effects for the following reasons. Each of its MOS transistors conducts the whole tail current, and no more than that, for half of the oscillation period, instead of having to deliver a short and intense current pulse for a small fraction of the same period. In this way, its behavior is much closer to the ideal case, both in terms of the resulting oscillation amplitude, as described by (64), and of the effective noise factor $\gamma$, since neither the overdrive voltage, nor the drain-source voltage need to be particularly large when both transistors are active, which is the only time when they do inject noise into the oscillator.
However, the above results apply strictly only to the ideal designs considered in this paper. An advantage of the real Colpitts oscillator is that it does not possess any parasitic node, and all parasitic capacitances can in principle be absorbed into the tank capacitances, if they are not too large (a severe problem is however that such capacitances are often nonlinear). In the $L C$-tank oscillator, on the other hand, the common-source node is a truly parasitic node, where a large parasitic capacitance would greatly boost the phase noise of the oscillator.

An analysis of the impact of this and other nonideal phenomena on the phase noise, in both the $1 / f^{2}$ and $1 / f^{3}$ phase noise regions, is beyond the scope of this work.

\section{MEASUREMENT ReSULTS}

Three differential Colpitts (with $n \approx 1 / 3$ ) and three $L C$-tank oscillators have been fabricated in a 4-M $0.35-\mu \mathrm{m}$ CMOS process with MIM capacitors and a $2-\mu \mathrm{m}$-thick top Aluminum metal layer for RF inductor design. The oscillators have been designed pairwise for three different center frequencies (2.6, 3.0, and $3.4 \mathrm{GHz}$, respectively), each pair displaying the same center frequency. The die photograph of the $3-\mathrm{GHz}$ oscillator pair is shown in Fig. 10. pMOS varactors working in the accumulation and depletion regions have been used to implement a $5 \%$ fine tuning, while a matrix of switchable MIM capacitors extends the overall tuning range to $15 \%$ with four overlapping bands. As mentioned in Section II-A1, the use of a center-tapped inductor has two purposes: to ensure a differential mode of oscillation, at the same time allowing for a very high maximum oscillation frequency, and to increase the resonator- $Q$ of the oscillator. The oscillators in each pair are built around the same inductor and the same fine-tuning varactor, which together dominate the overall losses of the passive components, thanks to the availability of high-quality MIM capacitors; in this way, a comparison between the performances of the $L C$-tank and the Colpitts oscillator in each should become both straightforward and robust. Unfortunately, the presence of the off-chip gate bias voltage in the Colpitts oscillator makes the comparison less clear-cut than anticipated, which will be discussed later.

Phase-noise measurements show that the phase noise tends to increase in all oscillators when the fine-tuning varactors are active, indicating that the amplitude-to-phase noise generation mechanism is not negligible (contrary to what was expected from simulations) even for the relatively small-area pMOS varactors adopted. Since our primary goal is to compare the intrinsic behavior of $L C$-tank versus Colpitts oscillator, we will in the following refer to measurements taken at the extreme of the fine-tuning band (i.e., for a varactor control voltage of $0 \mathrm{~V}$ ), where the varactor behaves like a linear capacitance.

For all three oscillator pairs and under a great variety of working conditions, the $L C$-tank oscillators display, compared to the Colpitts oscillators, a much lower phase noise. Defining as usual the phase-noise FoM as

$$
\operatorname{FoM}(\Delta \omega)=-\mathcal{L}(\Delta \omega)+20 \log \left(\frac{\omega_{0}}{\Delta \omega}\right)-10 \log (P)
$$

where $\omega_{0}$ is the angular frequency of oscillation, and $P$ is the power (in $\mathrm{mW}$ ) consumed by the oscillator, the FoM difference 

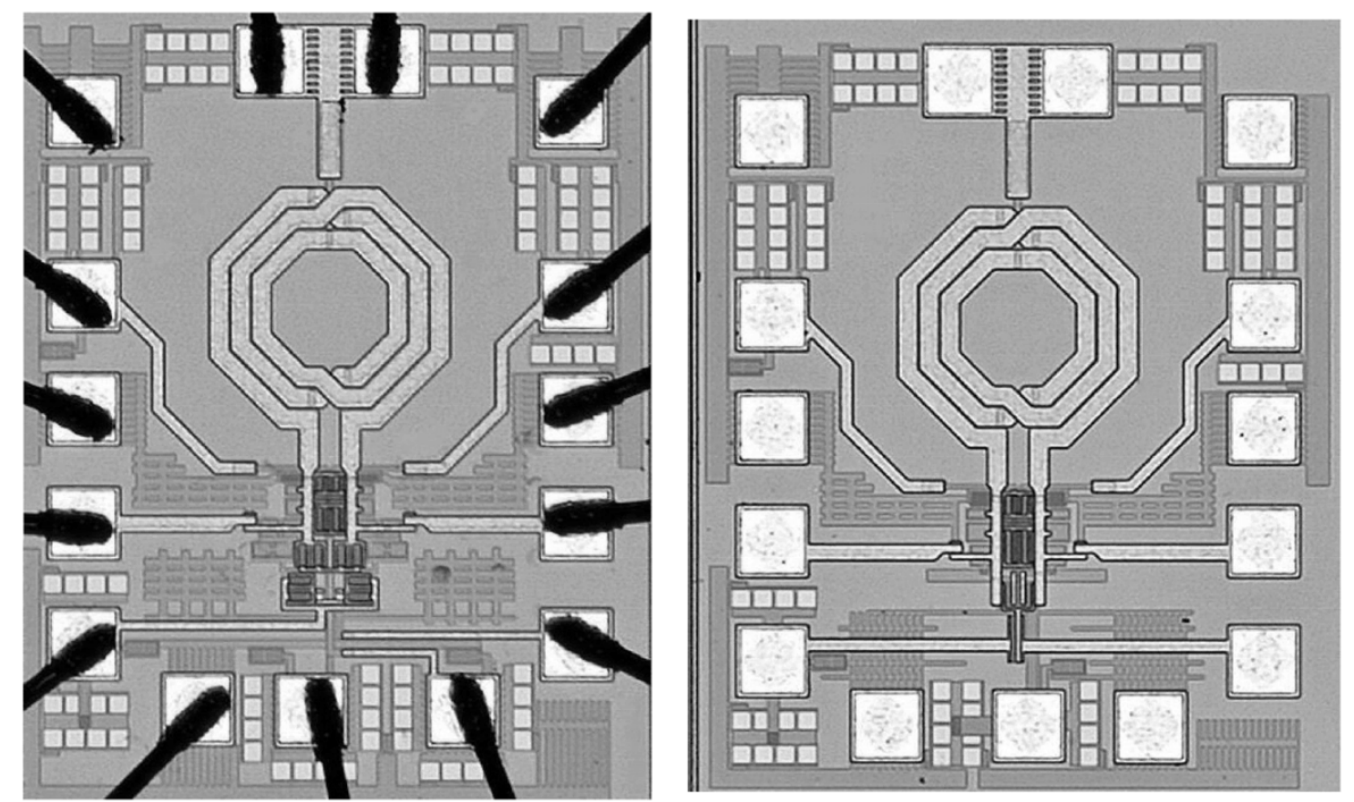

Fig. 10. Die photograph of (a) Colpitts and (b) LC-tank differential oscillators.

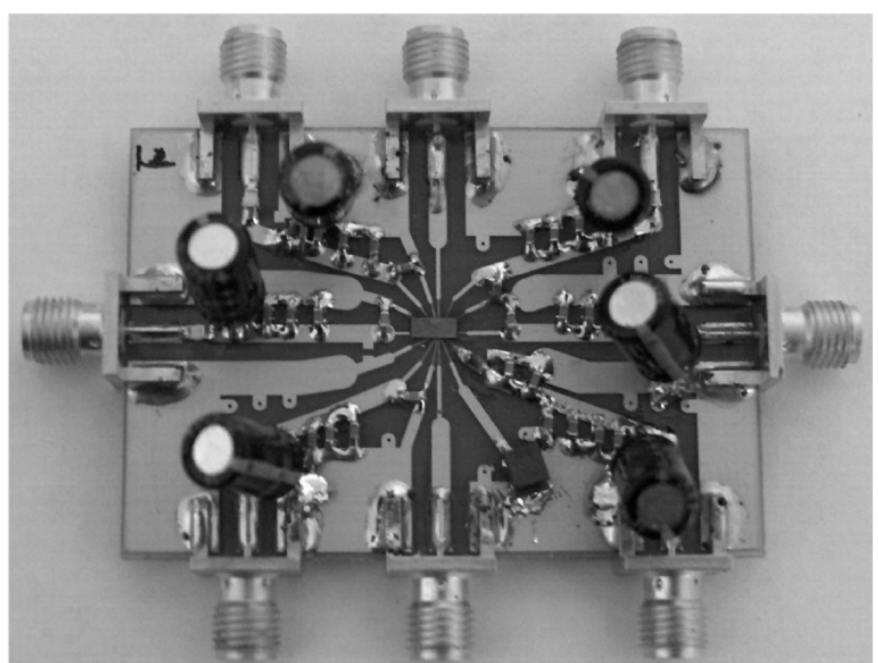

Fig. 11. Photograph of PCB with flipped, bump-soldered Colpitts chip.

varies between 6 and $8 \mathrm{~dB}$, always in favor of the $L C$-tank oscillator for each oscillator pair.

This is higher than what was expected from simulations, which suggested a maximum FoM difference of some $3 \mathrm{~dB}$. While it is always possible that some of the difference between simulations and measurements could be ascribed to deficiencies in the MOS model with the associated parameter values, a cause of systematic performance bias in favor of the $L C$-tank oscillators could actually be traced back to the parasitic components present at the gates of the transistors in the Colpitts oscillators [node $V_{B}$ in Fig. 1(b)]. In fact, through post-measurement simulations it was found that a $2-3-\mathrm{nH}$ parasitic bondwire inductance at the $V_{B}$ pad, in series with the (nonlinear) capacitive impedance seen at the gates of the MOS transistors, resonates at a frequency close to the oscillation frequency of the Colpitts oscillators. This spurious resonance has two effects: it reduces the amplitude of the desired oscillation, and boosts the losses

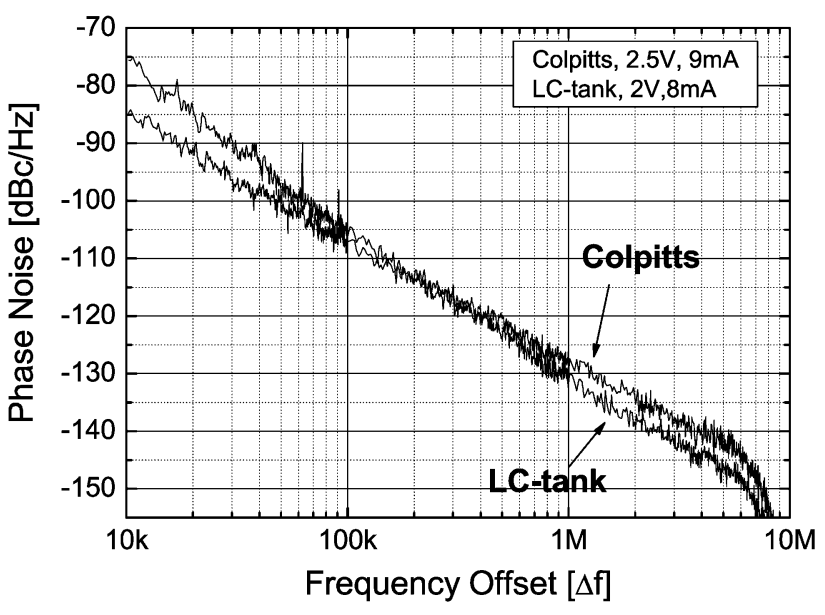

Fig. 12. Phase-noise measurements for Colpitts and $L C$-tank differential oscillators, for a carrier frequency of $2.9 \mathrm{GHz}$.

of the otherwise negligible parasitic interconnection resistance $(\approx 3 \Omega)$ between transistor gates and pad. In order to observe this deleterious phenomenon, both the $L C$-tank and the Colpitts oscillator in the 3-GHz pair were flipped and bump-soldered directly on the PCB (Fig. 11), thereby removing any uncertainty associated to the bondwire inductors and package parasitics. An off-chip resistance of a few tens of ohms, placed in series with $V_{B}$, prevented any resonance induced by the PCB parasitic inductance at the gates of the Colpitts transistors, while contributing a phase-noise increase of approximately $0.5 \mathrm{~dB}$.

Phase noise measurements taken on this oscillator pair showed that the $L C$-tank oscillator has in average a $5 \mathrm{~dB}$ higher FoM than the Colpitts oscillator. Compared to the previous measurements, this figure is considerably closer to what was expected from the theoretical analysis. As an example, Fig. 12 shows the phase noise plots for the oscillator pair for a carrier frequency of $2.9 \mathrm{GHz}$. The phase noise of the $L C$-tank oscillator consuming $8 \mathrm{~mA}$ from a $2-\mathrm{V}$ power supply is $-142.0 \mathrm{dBc} / \mathrm{Hz}$ at 
3-MHz offset, which yields a very high FoM of $\sim 189.5 \mathrm{dBc} / \mathrm{Hz}$. The Colpitts oscillator displays a phase noise of $-138.0 \mathrm{dBc} / \mathrm{Hz}$ for a power consumption of $2.5 \mathrm{~V} \times 9 \mathrm{~mA}$, which results in a FoM of $\sim 184.0 \mathrm{dBc} / \mathrm{Hz}$. As a last remark, it is interesting to note that the Colpitts oscillator consistently shows a lower $1 / \mathrm{f}$ noise upconversion into phase noise than the $L C$-tank oscillator.

\section{CONCLUSION}

This paper has shown that CMOS Colpitts and $L C$-tank oscillators are both capable of very good phase noise performances, achieved however through very different strategies for phase noise suppression: transistors in Colpitts oscillators generate a great amount of noise, but the conversion of this noise into phase noise is minimal; $L C$-tank transistors generate little noise due to source degeneration inside the oscillator, but all of this noise is converted into phase noise. Closed-form formulas for the phase noise of both oscillators have been derived and checked against spectreRF simulations. The analysis developed in this work, together with spectreRF simulations and measurement results taken on several oscillator prototypes, has shown that the $L C$-tank oscillator is superior to the Colpitts differential oscillator, at least as long as the phase noise performance in the $1 / f^{2}$ region is concerned.

\section{ACKNOWLEDGMENT}

The authors would like to thank Dr. Henrik Sjöland, Fredrik Tillman, and Niklas Troedsson at the Department of Electroscience, Lund University, Lund, Sweden, for helping taking all phase-noise measurements.

\section{REFERENCES}

[1] L. Dauphinee, M. Copeland, and P. Schvan, "A balanced $1.5 \mathrm{GHz}$ voltage controlled oscillator with an integrated $L C$ resonator," in IEEE ISSCC Dig. Tech. Papers, Feb. 1997, pp. 390-391.

[2] F. Mernyei, M. Pardoen, W. Höß, and F. Darrer, "Fully integrated RF VCO for wireless transceivers," in Proc. 1998 Int. Symp. Signals, Systems, and Electronics, Sep. 1998, pp. 79-82.

[3] D. Baek, S. Ko, J.-G. Kim, D.-W. Kim, and S. Hong, "Ku-band InGaPGaAs HBT MMIC VCO's with balanced and differential topologies," IEEE Trans. Microw. Theory Techn., vol. 52, no. 4, pp. 1353-1359, Apr. 2004.

[4] R. Aparicio and A. Hajimiri, "A noise-shifting differential Colpitts VCO,” IEEE J. Solid-State Circuits, vol. 37, no. 12, pp. 1728-1736, Dec. 2002.

[5] W. Michielsen, L.-R. Zheng, H. Tehnunen, S. Pinel, and J. Laskar, "Design considerations for a $2.4 \mathrm{GHz}$ differential Colpitts oscillator," in Proc. NORCHIP, Nov. 2003, pp. 232-235.

[6] A. Hajimiri and T. H. Lee, "A general theory of phase noise in electrical oscillators," IEEE J. Solid-State Circuits, vol. 33, no. 2, pp. 179-194, Feb. 1998.

[7] Q. Huang, "Phase noise to carrier ration in LC oscillators," IEEE Trans. Circuits Syst. I, Fundam. Theory Appl., vol. 47, no. 7, pp. 965-980, Jul. 2000.

[8] T. H. Lee, The Design of CMOS Radio-Frequency Integrated Circuits. Cambridge, U.K.: Cambridge Univ. Press, 1998.

[9] P. Andreani and X. Wang, "On the phase-noise and phase-error performances of multiphase LC CMOS VCOs," IEEE J. Solid-State Circuits, vol. 39, no. 11, pp. 1883-1893, Nov. 2004.
[10] A. Hajimiri and T. H. Lee, "Corrections to "A general theory of phase noise in electrical oscillators"," IEEE J. Solid-State Circuits, vol. 33, no. 6, p. 928, Jun. 1998.

[11] J. J. Rael and A. Abidi, "Physical processes of phase noise in differential LC oscillators," in Proc. IEEE Custom Integrated Circuits Conf., May 2000, pp. 569-572.

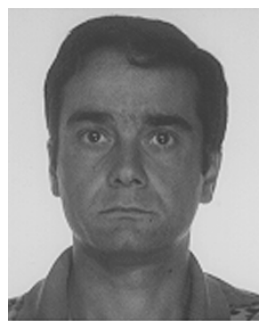

Pietro Andreani (S'98-A'99-M'01) received the M.S.E.E. degree from the University of Pisa, Italy, in 1988, and the Ph.D. degree from Lund University, Lund, Sweden, in 1999.

He joined the Department of Applied Electronics, Lund University, Sweden, in 1990, where he contributed to the development of software tools for digital ASIC design. During 1994, he was a CMOS IC Designer at the Department of Applied Electronics, University of Pisa, following which, he rejoined the Department of Applied Electronics, Lund University, as an Associate Professor, where he was responsible for the analog IC course package between 1995 and 2001. He is currently a Professor at the Center for Physical Electronics, Ørsted DTU, Technical University of Denmark, Kgs. Lyngby, Denmark, with analog/RF CMOS IC design as main research field.

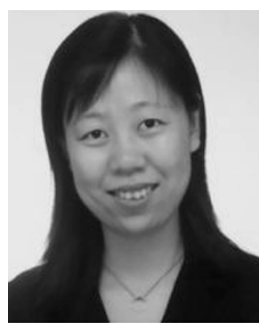

Xiaoyan Wang was born in China in 1976. She received the Bachelors degree from Nanjing University, China, in 1997, the Masters degree from the National University of Singapore in 2001, and the Ph.D. degree from the Technical University of Denmark, Kgs. Lyngby, in 2004.

Currently, she is working in the wireless LAN group at Infineon Technologies AG, Munich, Germany.

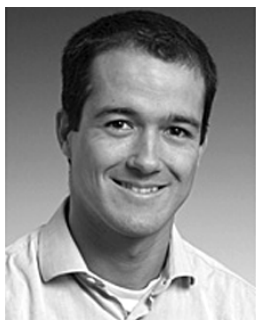

Luca Vandi was born in Ancona, Italy, in 1974. He received the M.S.E.E. degree in 2000 from the Politecnico di Milano, Italy.

He has been working with Nokia Mobile Phones, Copenhagen, Denmark, as an RF IC Design Engineer and System Engineer. Since June 2003, he has been working toward the Ph.D. degree at Ørsted DTU, Technical University of Denmark, Kgs. Lyngby, Denmark, where he is studying broadband solutions for RX front-ends.

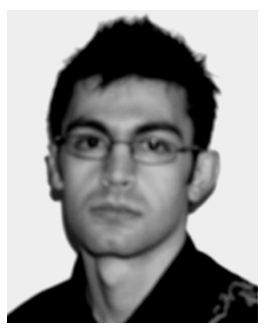

Ali Fard was born in Tehran, Iran, in 1978. He received the M.Sc. degree from Mälardalen University, Västerås, Sweden, in 2002.

In 2003, he joined the Department of Electronics, Mälardalen University, where he is currently working toward the Ph.D. degree on integrated wide-band RF VCOs. 